\title{
Advanced Model Based Control Studies for the Induction and Maintenance of Intravenous Anaesthesia
}

\author{
Ioana Nașcu, Alexandra Krieger, Clara M. Ionescu and Efstratios N. Pistikopoulos*
}

\begin{abstract}
The paper describes strategies towards modelbased automation of intravenous anaesthesia employing advanced control techniques. In particular, based on a detailed compartmental mathematical model featuring pharmacokinetic and pharmacodynamics information two alternative model predictive control strategies are presented: a model predictive control strategy, based on online optimisation, the Extended Predictive Self Adaptive Control (EPSAC) and a multiparametric control strategy based on offline optimisation, the multi-parametric model predictive control (mp-MPC). The multi-parametric features to account for the effect of nonlinearity and the impact of estimation are also described. The control strategies are tested on a set of 12 virtually generated patient models for the regulation of the depth of anaesthesia (DOA) by means of the Bispectral Index (BIS) using Propofol as the administrated anaestetic. The simulations show fast response, suitability of dose and robustness to induce and maintain the desired BIS setpoint.
\end{abstract}

Index Terms-Anaesthesia, inter-patient variability, MPC, EPSAC, mp-MPC, estimation

\section{INTRODUCTION}

A NAESTHESIA plays a very important role in surgery and in the intensive care unit. It is defined as a reversible pharmacological state of the patient where hypnosis, analgesia and muscle relaxation are guaranteed [1]. Analgesics block the sensation of pain; hypnotics produce unconsciousness, while muscle relaxants prevent unwanted movement of muscle tone.

The role of the anaesthetist has become more complex and indispensable to maintain the patients' vital functions before, during and after surgery. To estimate the drug effect in the patient's body and calculate the corresponding drug infusion rates average population models are used. These strategies may not always be safe for the patient since they do not take

This work was supported by the European Research Council (MOBILE, ERC Advanced Grant, No: 226462) and the European Commission (OPTICO/G.A. No.280813)

I. Nascu and A. Krieger are with the Centre of Process Engineering, Department of Chemical Engineering, Imperial College London, South Kensington Campus, London, SW7 2AZ, U. K. (e-mail: ioana.nascu@imperial.ac.uk, a.krieger@imperial.ac.uk)

C. Ionescu is with the Department of Electrical Energy, Systems and Automation, Ghent University, Gent, Belgium

*E. N. Pistikopoulos is with the Centre of Process Engineering, Department of Chemical Engineering, Imperial College London, (e-mail: e.pistikopoulos@imperial.ac.uk) and Artie McFerrin Department of Chemical Engineering, Texas A\&M, (e-mail: stratos@tamu.edu) into account any measured variable in a feedback control scheme and even if they reach the desired level of sedation fast, it can result in unsafe minimal values (undershoot) [1]. In stress situations the anaesthetist has to deal with routine assessments and simultaneously solve complex problems quickly. The automation of some routine actions of the anaesthetist can reduce the workload and consequently increase the safety of the patient.

The control of anaesthesia poses a manifold of challenges: inter- and intra-patient variability, multivariable characteristics, variable time delays, dynamics dependent on the hypnotic agent, model analysis variability, agent and stability issues [2], [3]. Hitherto, many PID tuning techniques have been elaborated. Since these classical controllers have no prior knowledge of the drug metabolism they cannot anticipate the response of the patient and their performance may be suboptimal. Other authors developed model based strategies using fuzzy [4], predictive [5], [6], [7], robust [8], [9], adaptive [2], [10] and multi-parametric MPC [11] control algorithms and applied them in clinical trials.

Drugs given for the induction and maintenance of DOA can be either inhalational or intravenous anaesthetics. An individualised physiological based, patient specific, compartmental model for volatile anaesthesia is presented and developed in [12] and a combined strategy of model predictive control (MPC) and estimation under uncertainty is presented in [13]. For intravenous anaesthesia, robustness tests of MPC for DOA using the EPSAC for a single input single output (SISO) model is presented in [14], different protocols for the administration of Propofol and Remifentanil (multiple input single output (MISO) model) are evaluated in [15] and in [16] a second output variable is determined, that originates from the effect of Remifetanil and leads to the implementation of a MIMO algorithm.

MPC is a model-based control technique that calculates the optimal control action considering constraints on the input, output and state variables by solving an optimization problem. The downside of this control technique is that the optimization problem has to be solved online. One way to avoid this is to use explicit/multi-parametric MPC which solves offline the optimization problem using multi-parametric programming and derives the control inputs as a set of explicit functions of the system states. An important advantage of mp-MPC is that the previously offline computed control laws can be easily implemented on embedded controllers. These types of devices use programming languages that cannot support powerful 
mathematical computations. The optimal control laws are retrievable immediately through simple function evaluations.

The aim of this paper is to design and compare four different types of model based controllers for administration of Propofol during ICU (Intensive Care Unit) sedation. Based on a compartmental pharmacokinetic (PK) and pharmacodynamic (PD) patient model, a predictive controller is first designed using an Extended Predictive Self Adaptive (EPSAC) strategy and three predictive controllers are designed using a mp-MPC strategy. The difference between the three controllers based on mp-MPC strategy is that one of them uses the linearized patient model whereas the other two use the compensation of the nonlinear part of the patient model. In one of the two controllers using the nonlinear compensation, the states are estimated using an online estimator, whilst for the other one the states are computed using the nominal patient model.

The paper is organized as follows: the patient model, the multi-parametric control strategy, the EPSAC strategy and the design of the controllers are presented in the following section. Section 3 presents the simulation results for the induction and maintenance phase and discussions are presented in Section 4. Finally Section 5 summarizes the main outcome of this paper.

\section{THEORETICAL BACKGROUND}

\section{A. Patient Model}

A compartmental model is used to describe the pharmacokinetic (PK) - pharmacodynamic (PD) blocks representing the distribution of drugs in the body, i.e. mass balance. The pharmacokinetic model represents the relation between the drug administration and drug concentration in the body, whereas the PD model represents the relation between the concentration of the drug in the central compartment and the effect observed on the patient. In each compartment the drug concentration is assumed to be uniform, as perfect and instantaneous mixing is assumed. The structure of the compartmental model is depicted in Fig. 1 [17], [18] .[10, 14]

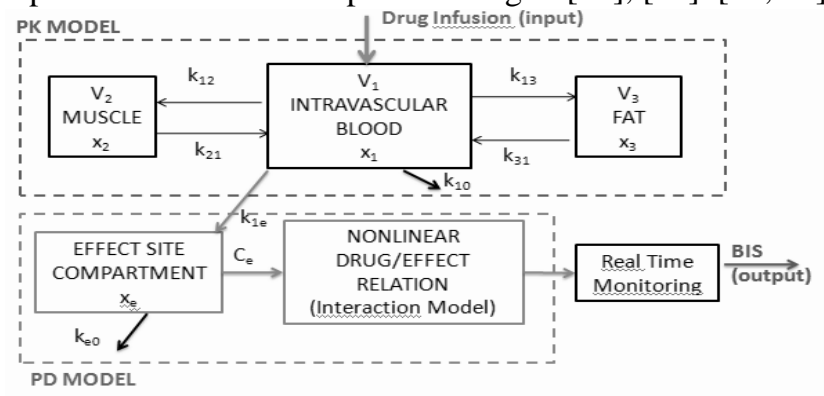

Fig. 1. Compartmental model of the patient, where PK denotes the pharmacokinetic model and PD denotes the pharmacodynamic model.

The PK-PD models most commonly used for Propofol are the $4^{\text {th }}$ order compartmental model described by Schnider [18], [19] and Minto [20], [21], respectively. These models, developed, tested and validated on a wide range of real patient data are commonly used in literature for the control of anaesthesia.

PK describes the distribution of the drug in the human body. The PK model and the first term of the PD model are considered linear studied on real patient data with the collaboration of anaesthesiologists and validated using blood samples provided by hospitals. [18, 19, 22]:

$$
\begin{aligned}
& \dot{x}_{1}(t)=-\left[k_{10}+k_{12}+k_{13}\right] \cdot x_{1}(t)+k_{21} \cdot x_{2}(t) \\
& +k_{31} \cdot x_{3}(t)+u(t) / V_{1} \\
& \dot{x}_{2}(t)=k_{12} \cdot x_{1}(t)-k_{21} \cdot x_{2}(t) \\
& \dot{x}_{3}(t)=k_{13} \cdot x_{1}(t)-k_{31} \cdot x_{3}(t) \\
& \dot{x}_{e}(t)=-k_{e 0} \cdot x_{e}(t)+k_{1 e} \cdot x_{1}(t)
\end{aligned}
$$

where $x_{1}$ represents the drug concentration in the central compartment [mg/l]. The peripheral compartments 2 (muscle) and 3 (fat) model the drug exchange of the blood with well and poorly diffused body tissues. The concentrations of drug in the fast and slow equilibrating peripheral compartments are denoted by $x_{2}$ and $x_{3}$ respectively. The parameters $k_{i j}$ for $i \neq j$, denote the drug transfer frequency from the $i^{\text {th }}$ to the $j^{\text {th }}$ compartment and $u(t)[\mathrm{mg} / \mathrm{min}]$ is the infusion rate of the anaesthetic or analgesic drug into the central compartment. The parameters $k_{i j}$ of the PK models depend on age, weight, height and gender and can be calculated for Propofol:

$$
\begin{aligned}
V_{1}= & 4.27[l], \mathrm{V}_{2}=18.9-0.391 \cdot(\text { age }-53)[l], \mathrm{V}_{3}=2.38[l] \\
\mathrm{C}_{11}= & 1.89+0.456(\text { weight }-77)-0.0681(l b m-59)+ \\
& +0.264(\text { height }-177)[l / \mathrm{min}] \\
\mathrm{C}_{12}= & 1.29-0.024(\text { age }-53)[l / \mathrm{min}], \mathrm{C}_{13}=0.836[l / \mathrm{min}] \\
\mathrm{k}_{10}= & \frac{C_{l 1}}{V_{1}}\left[\mathrm{~min}^{-1}\right], \mathrm{k}_{12}=\frac{C_{l 2}}{V_{1}}\left[\mathrm{~min}^{-1}\right], \mathrm{k}_{13}=\frac{C_{l 3}}{V_{1}}\left[\mathrm{~min}^{-1}\right], \\
\mathrm{k}_{21}= & \frac{C_{l 2}}{V_{2}}\left[\min ^{-1}\right], \mathrm{k}_{31}=\frac{C_{l 3}}{V_{3}}\left[\mathrm{~min}^{-1}\right], k_{e 0}=0.456\left[\mathrm{~min}^{-1}\right]
\end{aligned}
$$

where $C_{l l}$ is the rate at which the drug is cleared from the body, and $C_{l 2}$ and $C_{l 3}$ are the rates at which the drug is removed from the central compartment to the other two compartments by distribution.

The lean body mass $(\mathrm{lbm})$ for men $(\mathrm{m})$ and women (f) are calculated by:

$$
\begin{gathered}
l b m_{-} m=1.1 \cdot \text { weight }-128 \frac{\text { weight }^{2}}{\text { height }^{2}} \\
l b m_{-} f=1.07 \cdot \text { weight }-148 \frac{\text { weight }^{2}}{\text { height }^{2}}
\end{gathered}
$$

An additional hypothetical effect compartment is added to represent the lag between plasma drug concentration and drug response. The drug concentration in this compartment is represented by $x_{e}$, called the effect-site compartment concentration. The effect compartment receives drug from the central compartment by a first-order process and it is considered as a virtual additional compartment. Therefore, the drug transfer frequency for Propofol from the central compartment to the effect site-compartment is considered in clinical practice to be equal to the frequency of drug removal from the effect-site compartment $k_{e 0}=k_{l e}=0.456\left[\mathrm{~min}^{-1}\right.$ ] [18], [19], [23]. When considering the drug effect observed on the patient, the BIS variable can be related to the effect drug concentration $C_{e}$ by the empirical static nonlinear relationship [5], [6], [18], [19], [23], called also the Hill curve:

$$
B I S(t)=E_{0}-E_{\max } \cdot \frac{C_{e}(t)^{\gamma}}{C_{e}(t)^{\gamma}+E C_{50}^{\gamma}}
$$

Notice that in the model used in this paper $C_{e}=x_{e}$. 
$E_{0}$ denotes the baseline value (awake state - without drug), which by convention is typically assigned a value of $100, E_{\max }$ denotes the maximum effect achieved by the drug infusion, $E C_{50}$ is the drug concentration at $50 \%$ of the maximal effect and represents the patient sensitivity to the drug, and $\gamma$ determines the steepness of the curve.

The inverse of the Hill curve can be defined by the following formulation:

$$
C_{e}(t)=E C_{50}\left(\frac{E_{0}-B I S(t)}{E_{\max }-E_{0}+B I S(t)}\right)^{\frac{1}{\gamma}}
$$

The type of models that consider a linear dynamic followed by a nonlinear dynamic system are called WienerHammarstein models and are presented in Fig. 2. These type of models have been widely used in control of anaesthesia [23]. For the automatic regulation of depth of anaesthesia (DOA) in Fig. 2 the anaesthetic agent, i.e. Propofol, is the input and the Bispectral Index (BIS) the output of the system. Because of its pharmacological profile, Propofol is applicable for both induction and maintenance of hypnosis during anaesthesia and intensive care sedation [24].

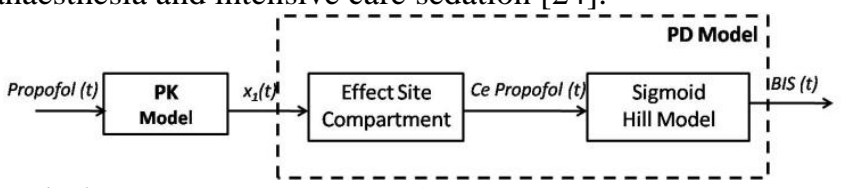

Fig. 2. Schematic representation of the NONLINEAR SISO patient model for intravenous anaesthesia

The Bispectral Index (BIS) is a signal that is derived from the electro-encephalogram (EEG) used to assess the level of consciousness in anaesthesia. A BIS value of 0 equals EEG silence, while a BIS value of 100 is the expected value of a fully conscious adult patient, 60-70 and 40-60 range represents light and moderate hypnotic conditions, respectively. The target value during surgery is 50 , giving us a gap between 40 and 60 to guarantee adequate sedation [1], [2], [3].

\section{B. Advanced Model Based Control Strategies}

MPC is a control methodology based on two main principles: explicit on-line use of a process model to predict the process output at future time instants and the computation of an optimal control action by minimizing one or more cost functions, including constraints on the process variables.

The main differences between the different types of MPC algorithms are: the type of model used to represent the process and its disturbances and the cost function(s) to be minimized, with or without constraints.

\section{1) EPSAC Strategy}

For the EPSAC approach, described in detail in [25], the controller output is obtained by minimizing the cost function:

$$
\sum_{k=N_{1}}^{N_{2}}[r(t+k / t)-y(t+k / t)]^{2}+\lambda \sum_{k=0}^{N_{u}-1}[\Delta u(t+k / t)]^{2}(6)
$$

The design parameters are: $N_{l}=$ the minimum costing horizon $N_{2}=$ the maximum costing horizon, $N_{2}-N_{1}=$ the prediction horizon $N_{\mathrm{u}}=$ control horizon, $\lambda$ =weight parameter, $n(t)$ is the disturbance, $y(t)$ the measured output and $u(t)$ the model input. The signal $r$ represents the reference trajectory.

In our case the process input is represented by the Propofol infusion rate applied to the patient. The process output is predicted at time instant t over the prediction horizon $N_{2}-N_{1}$, based on the measurements available at that moment and the future outputs of the control signal. The cost function is an extended EPSAC cost function that penalizes the control movements using the weight parameter $\lambda$.

\section{2) Multi-Parametric Strategy}

Multi-parametric programming is a technique to solve an optimization problem, where the objective is to minimize or maximize a performance criterion subject to a given set of constraints where some of the parameters vary between specified lower and upper bounds. The main characteristic of mp-MPC is its ability to obtain: (i) the objective and optimization variable as a function of the varying parameters, and (ii) the regions in the space of the parameters where these functions are valid (critical regions) [26, 27] .This reduces the online implementation of the MPC to simple function evaluation, facilitating real time applications.

For the mp-MPC, the generic optimization problem solved is:

$$
\begin{gathered}
\min _{x, y, u} J=x_{N}^{\prime} P x_{N}+\sum_{k=1}^{N-1} x_{k} Q_{k} x_{k}+\sum_{k=1}^{N-1}\left(y_{k}-y_{k}^{R}\right)^{\prime} Q R_{k}\left(y_{k}-y_{k}^{R}\right)+ \\
+\sum_{k=0}^{N_{u}-1}\left(u_{k}-u^{R}\right)^{\prime} R_{k}\left(u_{k}-u^{R}\right)+\sum_{k=0}^{N_{u}-1} \Delta u_{k} R 1_{k} \Delta u_{k} \\
\text { s.t. } \mathrm{x}_{\mathrm{t}+1}=A x_{t}+B u_{t} \\
\mathrm{y}_{\mathrm{t}}=C x_{t} \\
\mathrm{BIS}_{\min } \leq y \leq B I S_{\max } \\
\Delta \mathrm{u}_{\text {min }} \leq \Delta u \leq \Delta u_{\max } \\
x_{t} \in X \subseteq \mathfrak{R}^{p}, \boldsymbol{u}_{t} \in U \subseteq \mathfrak{R}^{s}
\end{gathered}
$$

where $x$ are states, $y$ outputs and $u$ controls, all (discrete) time dependent vectors. The subsets of output variables that get tracked have time-dependent set points $y^{R}$. Finally $\Delta \mathrm{u}$ are changes in control variables, $\Delta u(k)=u(k)-u(k-1)$. The prediction horizon is denoted by $N$ and control horizon by $N_{u}$. $X, U$ are the sets of the state and input constraints that contain the origin in their interior. Both $Q>0$, the objective coefficient for the states and $P>0$, the terminal weight matrix for the states, are symmetric semi-positive definite matrices. The quadratic matrix for manipulated variables $R>0$ is a symmetric positive matrix, $Q R$ is the quadratic matrix for tracked outputs and $R l$ is a weight matrix for the control action changes $(\Delta u)$. The control problem is posed as a quadratic convex optimization problem for which an explicit solution can be obtained as follows:

$$
u=f(x)=\left\{\begin{aligned}
K_{1} x+c_{1} & \text { if } x \in C R^{1} \\
& \cdots \\
K_{s} x+c_{s} & \text { if } x \in C R^{s}
\end{aligned}\right.
$$

where $s$ is the number of critical regions.

\section{Control Design}

The presence of the Hill nonlinearity complicates the use of linear controller synthesis. Two methods to overcome this problem have been proposed: exact and local linearization. Exact linearization is based on the compensation of the nonlinearity introduced by the Hill curve, in the PD model. Since the Hill nonlinearity (4) is a monotonic function $(f)$ of the normalized effect site concentration, it has an inverse presented in (5). Using a parameter scheduling technique the inverse Hill function $\left(f^{1}\right)$ could be implemented in the 
controller as illustrated by the block diagram in Fig. 3. Here $f$ is using the nonlinearity parameter of the real patient $\left(E_{0}\right.$, $E_{\max }, E C_{50}, \gamma$ ), while $f^{l}$ is using the parameter assumed by the controller (the nominal patient nonlinearity parameters a priori known $\left(E_{0}^{\text {mean }}, E_{\max }{ }^{\text {mean }}, E C_{50}{ }^{\text {mean }}, \gamma^{\text {mean }}\right)$. The controller aims at controlling the estimated drug concentration $\hat{C}_{e}$, which is straight-forward, using a linear controller.

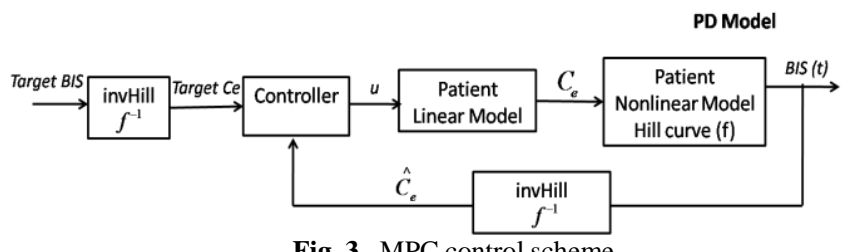

An exact linearization occurs only in the case where the patient model is identical to the nominal model in which case it completely cancels the nonlinearity and $\hat{C}_{e}=C_{e}$. The local linearization is based on the linearized PK-PD model for a BIS value of 50 obtained using gPROMS [28]

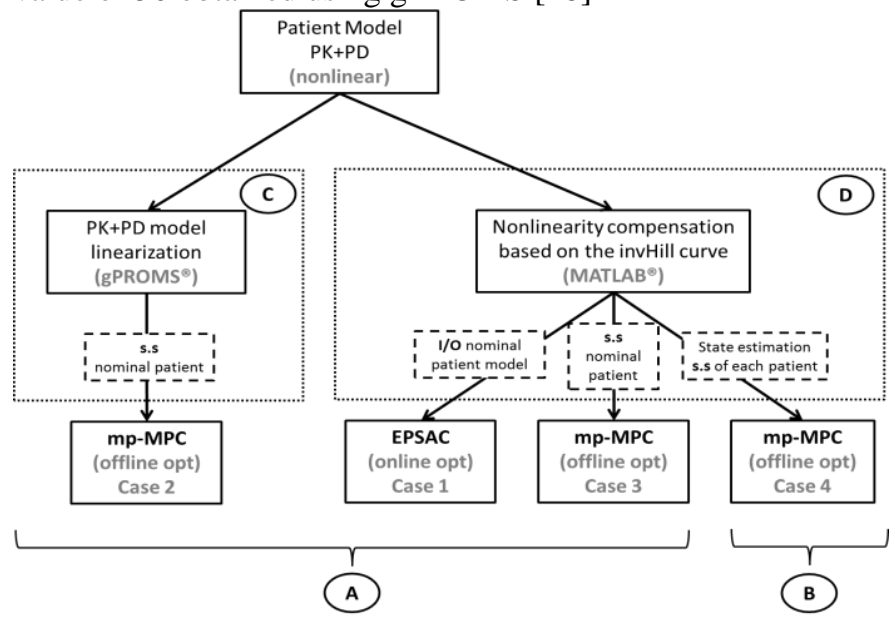

Fig. 4. Control scheme development flowchart

An important challenges of DOA control is the high interand intra- patient variability. This results in different dynamics in PK model, and changes in the parameters of the Hill function for each patient model. Four control strategies, a model predictive controller, Extended Predictive Self Adaptive Controller (EPSAC), and three different multiparametric model predictive controllers (mp-MPC) are designed and evaluated. The framework for the different ways of designing the controllers is presented in Fig. 4.

The patient response is simulated using Patient model block, composed of the PK-PD linear part (1) and the nonlinear PD part, the Hill nonlinearity (4). BIS can be measured, however the states cannot and have to be estimated: either using the drug rate and the nominal state space patient model, or by using the input and output measured output (BIS) of the process, the state space nominal model and a correction estimator based on the output changes.

To analyse the influence of the changes in the dynamics of the PK model on the control performances, two types of control schemes are implemented, one uses the states given by the nominal model (B) and the other uses an estimator to adjust the states based on the dynamics of each patient (A).

The influence to the changes of parameters of the Hill curve on the control performances is analysed by two types of control schemes, one using the local linearized PK-PD model (C) and the second is based on the exact linearization (D). The following design parameters are used: the objective coefficients for states $(x), Q=0$ when we have no state estimation and $\mathrm{Q}=1$ in the case with state estimation, the quadratic matrix for tracked outputs $(y), Q R=1000$, quadratic matrix for manipulated variables $(u), R=1$, the control horizon $N_{u}=1$ and the prediction horizon $N=20$ in both mp-MPC and EPSAC. The EPSAC has an extra weighting factor $\lambda$ from (8) for which its default value $\lambda=0$ was used. The states used in the design of the controllers are $x_{1}, x_{2}, x_{3}, x_{e}$ as described in (1). The clinically recommended sampling time is of 5 seconds [6]. $\mathrm{N}_{1}, \mathrm{~N}_{2}$ and $\mathrm{N}_{\mathrm{u}}$ are chosen based on the characteristics of the process and the desired performances. Based on [29, 30] $\mathrm{N}$ should be large, at least $2 \mathrm{n}-1$ but no larger than the rise-time of the process. For anaesthesia due to medical procedures we are constrained to use a small sampling time leads to a choice of a greater value for N. Also, the dead time is not considered since it's very small and does not affect the process, therefore $\mathrm{N}_{1}=1$. In choosing $\mathrm{N}_{\mathrm{u}}$, for processes with no unstable/underdamped poles, like anaesthesia, $\mathrm{Nu}=1$ is generally satisfactory. A choice of the Q, R and QR is given by Bryson's rule [31].

1) Case 1: EPSAC

In this section we apply a particular case of online MPC, the EPSAC (Extended Prediction Self-Adaptive Control) strategy described in detail in Section 2.3. The structure of the control system proposed in this section is shown in Fig. 5.

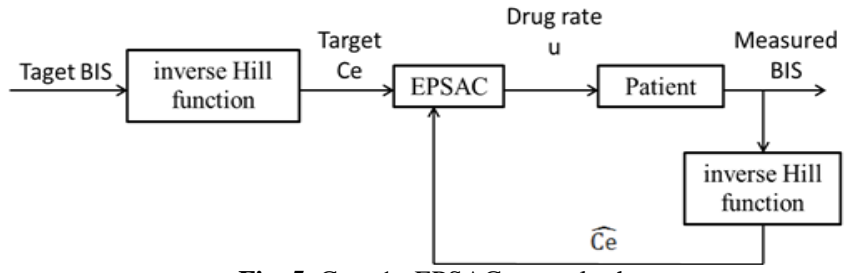

Fig. 5. Case 1: EPSAC control scheme

The Patient block is composed of the PK and PD models. Control strategy based on nonlinearity compensation and I/O linear nominal patient model is used (Fig.4) The controller output is obtained by minimizing cost function (6) with the design parameters in section II.B.1 The control algorithm uses for prediction a transfer function derived from the PK-PD linear model (1). The inverse of the Hill curve (5) is used to compensate the nonlinearity. Both the linear model and the inverse of the Hill curve use the nominal values from Table 1.

2) Case 2: $m p-M P C$ without nonlinearity compensation

The structure of the control scheme is presented in Fig.6. This approach uses the explicit/multi-parametric Model Predictive Control strategy based on local linearization of the PK-PD model and the state space model of the linearized nominal patient model (Fig.4).

To obtain the linearized patient model we will first implement the PK and PD model for the nominal patient in gPROMS [28] and determine the state space of the linearized nominal patient model at $\mathrm{BIS}=50$. Using these matrices the mp-QP optimization problem (7) is solved to obtain the critical regions CR, using a Matlab implementation of multiparametric quadratic programming algorithm [32] and determine the mp-MPC controller. 


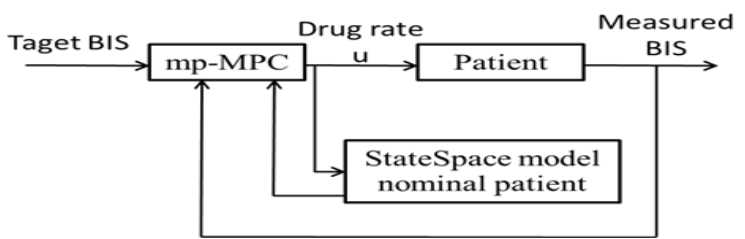

Fig. 6. Case 2: mp-MPC without nonlinearity compensation - control scheme 3) Case 3: $\mathrm{mp}-\mathrm{MPC}$ with nonlinearity compensation

The explicit/multi-parametric Model Predictive Control is used again. Control strategy based on nonlinearity compensation and the state space model of the PK-PD linear part (1) for the nominal patient model is used (Fig.4). The PKPD model is no longer linearized as a whole in gPROMS like in the previous case (Case2). Instead, the PK-PD linear part (1), is implemented in Matlab and is used to obtain the state space of the nominal patient characteristics (A, B, C and D matrices). Having the state space obtained we solve the $\mathrm{mp}$ QP optimization problem (7), obtain the (CR) using POP [32] and determine the controller based on the nominal patient values.

The inverse of the Hill curve (5) based on the nominal patient model parameters is then used to compensate the nonlinearity. Note that the states are obtained using the state space model based on the A, B, C and D matrices and the drug rate $\mathrm{u}$ as input. This control scheme is presented in Fig. 7.

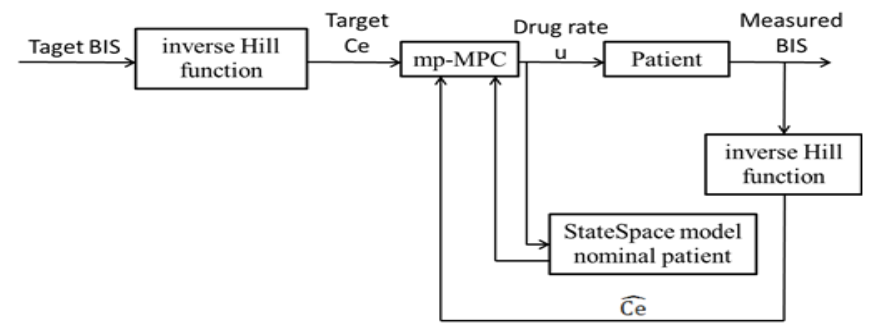

Fig. 7. Case 3:mp-MPC with nonlinearity compensation - control scheme 4) Case 4: $m p-M P C$ with nonlinearity compensation and estimation

This approach also uses the explicit/multi-parametric Model Predictive Control strategy. The structure of this control scheme is similar to the one described in II.C.3.

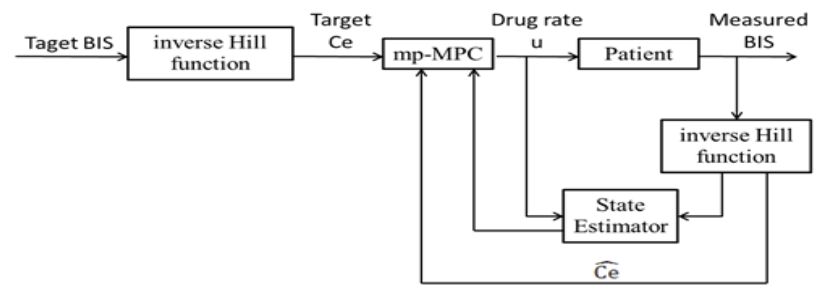

Fig. 8. Case 4: mp-MPC with nonlinearity compensation and estimator control scheme

The difference is that the State Space model nominal patient block from Fig.7 is replaced by a State Estimator. Here, the real patient states are estimated using a Kalman filter [33] based on the state space of the nominal patient, the online BIS measurement and the drug rate.

\section{RESULTS}

In this section the results of a simulation study to evaluate the four control strategies, for the administration of Propofol are presented. DOA is monitored using the Bispectral Index (BIS) during the induction and maintenance phase of general anaesthesia. The closed loop control tests are performed on a set of 12 patients [14] plus an extra patient representing the nominal values of all 12 patients ( $\mathrm{PaN}$ - patient nominal). The parameters values of these patients are given in Table 1 and are also used to calculate the parameters of the patient model.

TABLE 1

BIOMETRIC VALUES OF THE VIRTUAL PATIENTS

\begin{tabular}{|c|c|c|c|c|c|c|c|}
\hline ت & $\underset{4}{*}$ & 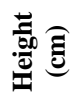 & 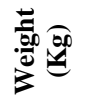 & Uूँ & $\sum_{\text {In }}^{00}$ & 도 & $>$ \\
\hline 1 & 40 & 163 & 54 & $\mathrm{M}$ & 6.33 & 98.8 & 2.24 \\
\hline 2 & 36 & 163 & 50 & $\mathrm{M}$ & 6.76 & 98.6 & 4.29 \\
\hline 3 & 28 & 164 & 52 & M & 8.44 & 91.2 & 4.1 \\
\hline 4 & 50 & 163 & 83 & M & 6.44 & 95.9 & 2.18 \\
\hline 5 & 28 & 164 & 60 & $\mathrm{~F}$ & 4.93 & 94.7 & 2.46 \\
\hline 6 & 43 & 163 & 59 & $\mathrm{M}$ & 12.0 & 90.2 & 2.42 \\
\hline 7 & 37 & 187 & 75 & $\mathrm{~F}$ & 8.02 & 92.0 & 2.1 \\
\hline 8 & 38 & 174 & 80 & M & 6.56 & 95.5 & 4.12 \\
\hline 9 & 41 & 170 & 70 & M & 6.15 & 89.2 & 6.89 \\
\hline 10 & 37 & 167 & 58 & M & 13.7 & 83.1 & 1.65 \\
\hline 11 & 42 & 179 & 78 & $\mathrm{~F}$ & 4.82 & 91.8 & 1.85 \\
\hline 12 & 34 & 172 & 58 & $\mathrm{M}$ & 4.95 & 96.2 & 1.84 \\
\hline Mean & 38 & $\begin{array}{l}169 \\
\end{array}$ & 65 & $\mathrm{M}$ & 7.42 & 93.1 & 3 \\
\hline
\end{tabular}

All designed controllers are simulated first for the set of data presented in Table 1 in order to have a better understanding of their behaviour on the different types of patients, and analyse the inter- and intra- patient variability. Next, the four controllers will be tested against each other and simulated for different patients so as to be able to compare their performances by means of the BIS index and the corresponding Propofol infusion rates.

\section{A. Induction Phase}

Ideally the induction phase of the patient in an operational DOA is performed as fast as possible, such that little time is lost before the surgeon can start operating. It is therefore desirable that the patient reaches the $\mathrm{BIS}=50$ target and remains within the target value without much undershoot or overshoot, i.e. values below $\mathrm{BIS}=40$ and above $\mathrm{BIS}=60$ should be avoided. In Fig.9, Fig. 11, Fig. 13, Fig. 14 we have the simulations of the four controllers for all 12 patients and the nominal one in the induction phase. Fig. 10 presents the map of the critical region for the controller using local linearization (Case 2) and in Fig. 12 we have the map of the critical regions for the controllers designed using exact linearization, by using the inverse of the Hill curve (Case 3 and 4).

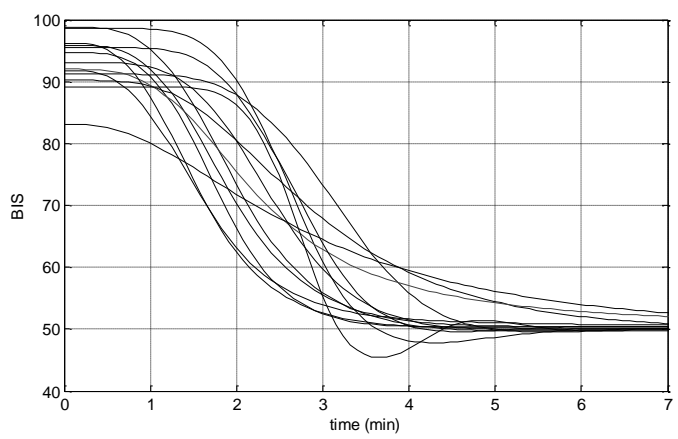

Fig.9. BIS output for all 13 patients for Case 1 


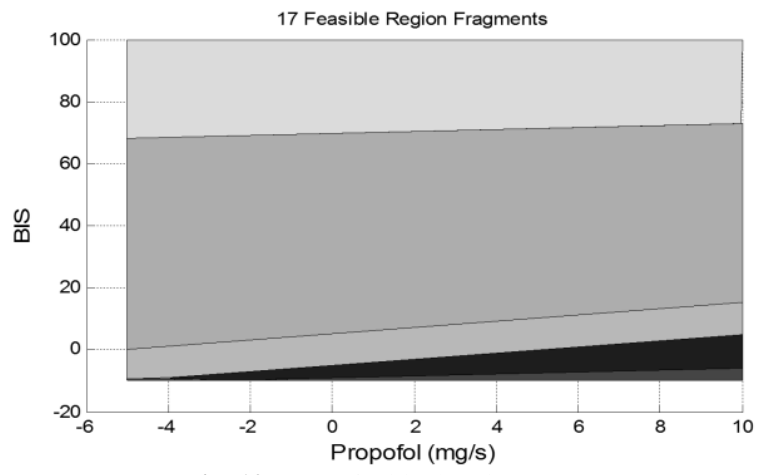

Fig. 10. Map of critical regions Case 2

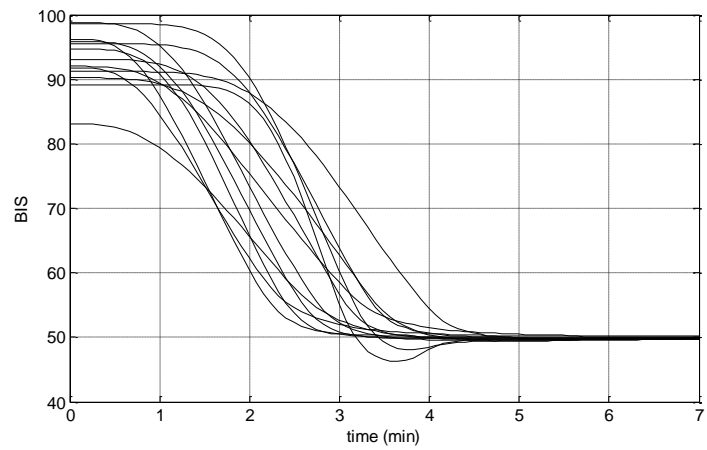

Fig. 11. BIS output for all 13 patients for Case 2

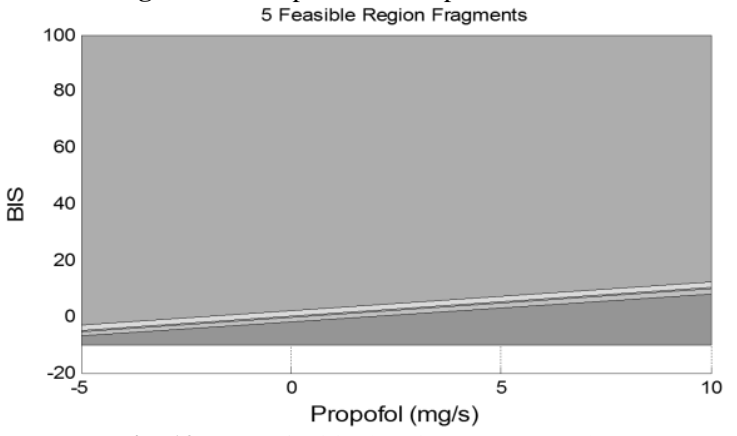

Fig. 12. Map of critical regions Case 3 and Case 4

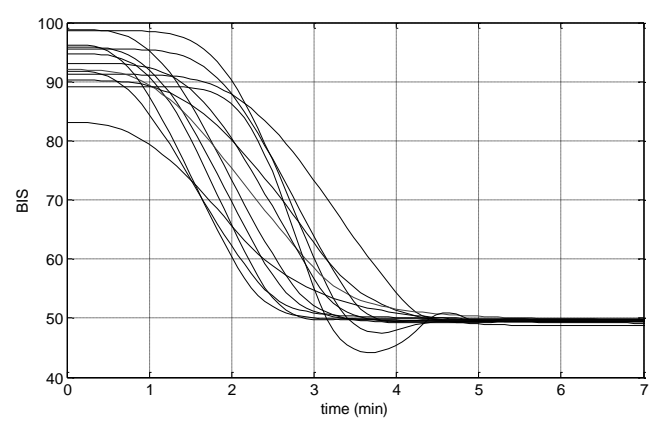

Fig. 13. BIS output for all 13 patients for Case 3

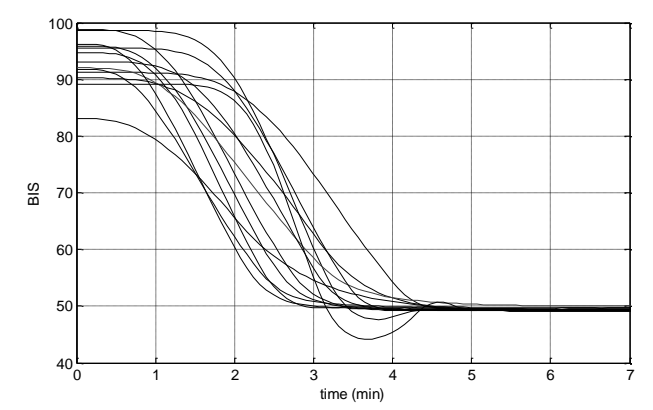

Fig. 14. BIS output for all 13 patients for Case 4
Simulations of some patients show very small oscillations around the steady state values. The average settling time for EPSAC is approximately $7 \mathrm{~min}$ and for the mp-MPC controllers is approximately $5 \mathrm{~min}$. In common practice the operation procedure does not start until the patient reaches an adequate DOA, usually taking up to $15 \mathrm{~min}$. Thus, a rise time between $5 \mathrm{~min}$ and $7 \mathrm{~min}$ gives good performances.

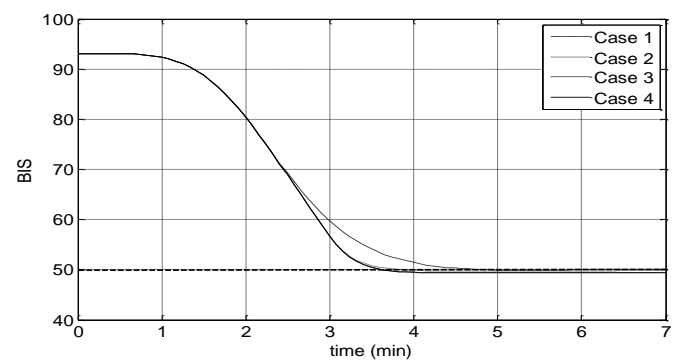

Fig. 15. BIS response for the four controllers for $\mathrm{PaN}$

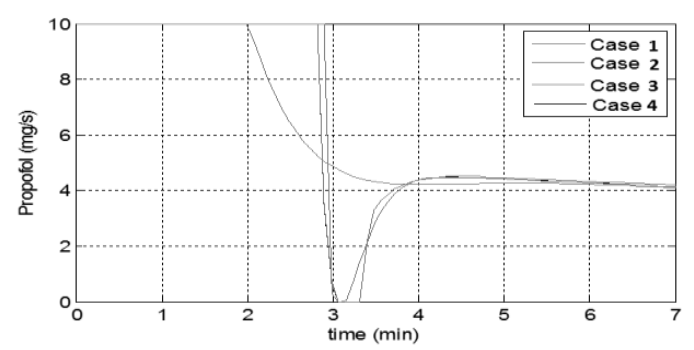

Fig. 16. Output for the four controllers for the PaN

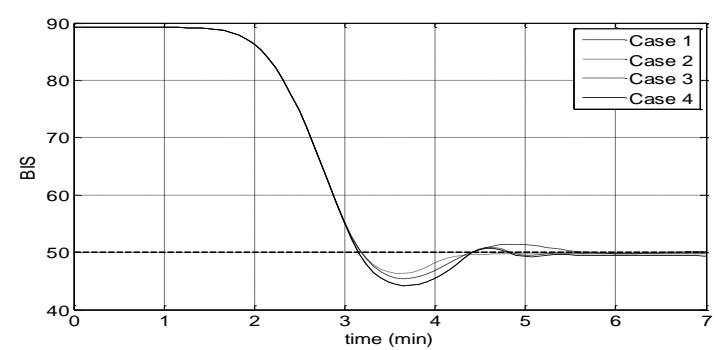

Fig. 17. BIS response for the four controllers for patient 9

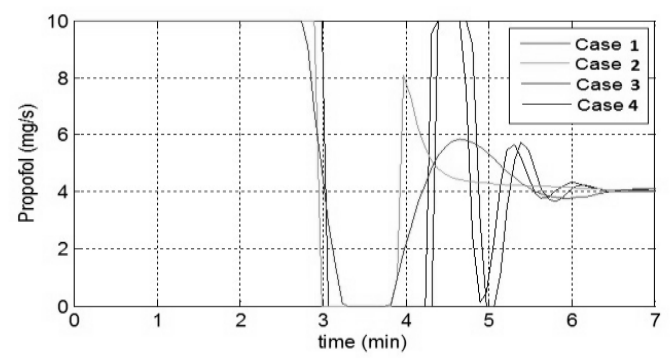

Fig. 18. Output for the four controllers for patient 9

The best performances are obtained for Case 2. It seems that the local linearization is able to deal with inter- and intrapatient variability Also, the process was linearized at $\mathrm{BIS}=50$ which is the value of the controller set point. The EPSAC controller is more influenced by inter-patient variability and for some patients the settling time has greater values.

The four controllers: EPSAC and the mp-MPC controllers, are simulated, compared for PaN and presented in Fig. 15. For patient 9, the most sensitive patient, this simulation is presented in Fig. 17. In Fig. 16 and Fig. 18 we have the corresponding Propofol infusion rates for the two patients. We can observe that due to the less aggressive behaviour of the EPSAC controller the output evolution will be smoother. In all 
the cases, the Propofol infusion rates are limited to $10 \mathrm{mg} / \mathrm{s}$ due to pump restrictions. The same conclusions as for Fig 9-12 are valid here. For both simulated patients the EPSAC controller has a slower response.

\section{B. Maintenance Phase}

During the maintenance phase, it is important that the controller rejects the disturbances occurred during surgery as fast as possible and bring the patient to the BIS target value. In this phase, typical disturbances can be applied additively to the output of the process to check the controller's ability to reject them [22]. A standard stimulus profile is defined and is presented in Fig 17. Each interval denotes a specific event in the operation theatre. Stimulus A represents response to intubation; B a surgical incision that is followed by a period of no surgical stimulation (i.e. waiting for pathology result); C mimics an abrupt stimulus after a period of low level stimulation; D the onset of a continuous normal surgical stimulation; E, F, and $\mathrm{G}$ simulate short-lasting, larger stimulation within the surgical period; and $\mathrm{H}$ represents the withdrawal of stimulation during the closing period [34].

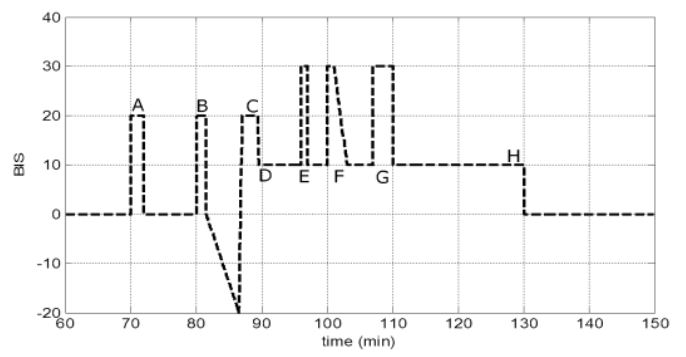

Fig. 19. The artificially generated disturbance signal

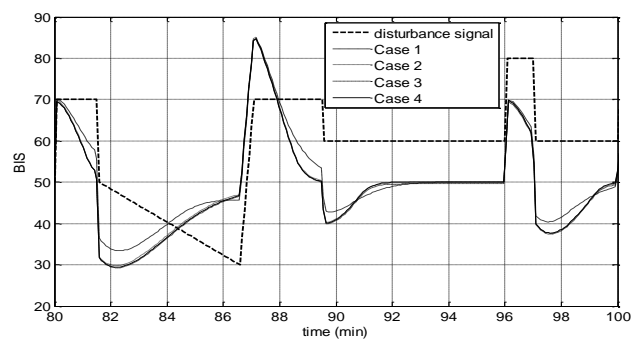

Fig. 20. BIS response for the four controllers for $\mathrm{PaN}$ with disturbance

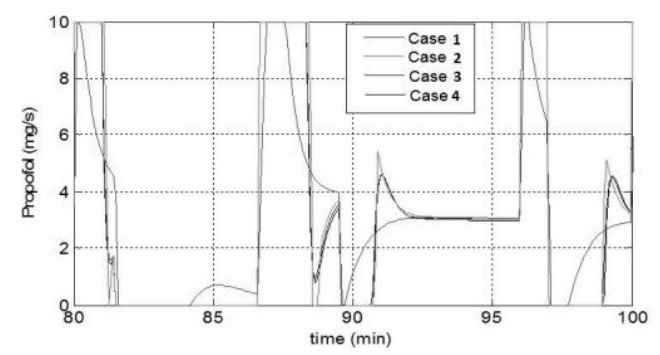

Fig. 21. Output for the four controllers for PaN with disturbance

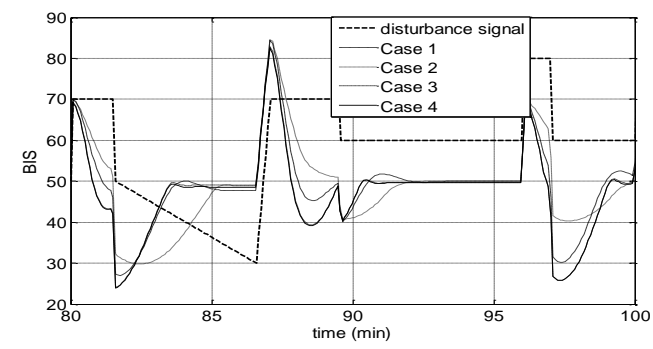

Fig. 22. BIS response for the four controllers for patient 9 with disturbance

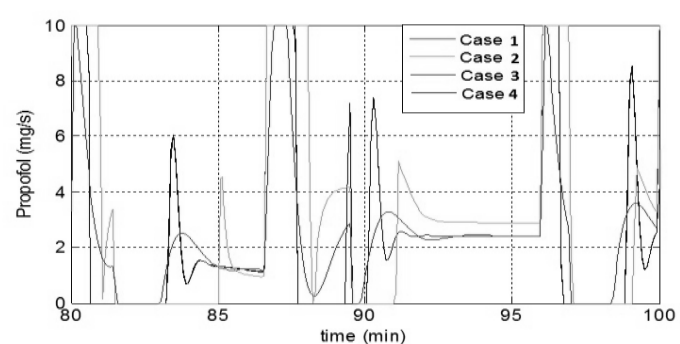

Fig.23. Output for the four controllers for patient 9 with disturbance

In Fig. 20 and Fig. 22 the performance of disturbance rejection of the four controllers for $\mathrm{PaN}$ and a more sensitive patient (patient 9) are shown. The figures present the most challenging part of the disturbance rejection test, namely B-CD-E. In Fig. 21 and Fig.23 we have the corresponding Propofol infusion rate for $\mathrm{PaN}$ and patient 9, limited between 0 and $10 \mathrm{mg} / \mathrm{s}$. The simulations are performed for the maintenance phase using the disturbance signal Fig. 19, between 60 minutes and 140 . The simulations show only small differences between the controllers and thus comparable performances of all four controllers. For the second control scheme the behaviour of the controller is less aggressive, the response is slower but it also has the smallest values of the undershoot.

\section{DISCUSSION}

The aim of this study is to evaluate the performance of a model based predictive control algorithm and model predictive multi-parametric control for automatic induction and control of depth of anaesthesia (DOA) during the induction and the maintenance phases.

Some of the most important aspects of this application are the high inter- and intra- patient variability, variable time delays, dynamics dependent on the hypnotic agent, model analysis variability. These are just some of the issues that are dealt with when trying to control the DOA.

In this paper the hypnotic agent Propofol is given as input and the output is described by the BIS, resulting in a SISO system. SISO patient models for control of most anesthetic drugs already exist in the literature and their parameters are estimated based on age, weight, gender and height.

Four different types of controllers are designed and tested. The first controller is based on the online optimization EPSAC MPC technique. The other three controllers are based on the offline optimization mp-MPC: one uses the linearized patient model and the other two use the compensation of the nonlinear part of the patient model. The difference between the two control strategies using nonlinearity compensation is that for one of them the states are computed using the nominal patient model whereas the other one uses an online estimator.

In order to address the issue of inter- and intra- patient variability, each of the four controllers are first tested for the whole set of patients presented in Table 1 for the induction and the maintenance phase. The maps of the critical regions for the mp-MPC are presented in Figures 10 and 12. One can observe that for the controllers using the nonlinearity compensation (exact linearization) there are less critical regions than for the controller using local linearization. This will make the controllers from Case 3 and 4 easier to implement on embedded devices. 
In the induction phase, for Case 1, representing the online EPSAC controller, we have an average settling time of 390 seconds. The undershoot of the most sensitive patient is of 4.6\%. As it can be observed from Fig. 11, Fig. 13 and Fig. 14, representing the BIS response of the mp-MPC controllers, the three cases have very similar settling time, lower than for the EPSAC strategy, an average of 270 seconds. For the undershoot evaluation the worst case scenario is considered, meaning the most sensitive patient. We obtain for the first controller (Case 2) an undershoot of $3.7 \%$, for Case 3 an undershoot of 5.8\% and for Case $45.78 \%$. All undershoots are below $10 \%$ which represents the maximum limit. For the induction phase it can be said that all four controllers perform well each of them having their own advantages: i.e. lower settling time, smaller undershoot.

The controllers are tested in the maintenance phase in order to see how well they can deal with disturbance rejection. In Fig. 20 and Fig. 22 we can observe the four controllers response to a disturbance signal that mimics the events that occur in an operation theatre for $\mathrm{PaN}$ and for patient 9.

All four controllers are tested against each other for the induction and maintenance phase for two different patients. The first patient is $\mathrm{PaN}$, and the second patient used for comparison, patient 9, represents the most sensitive patient. It is worth mentioning that the controllers are designed using the values of the nominal patient which means that for this patient we will have the best behaviour of the controllers. As it can be observed from Fig. 15, Fig. 16, Fig. 20 and Fig. 21, the BIS response and the output for $\mathrm{PaN}$ in the induction phase and the maintenance phase respectively, the three offline controllers have a very similar behaviour. All the controllers present no undershoot and a fast settling time. The EPSAC controller has a less aggressive behaviour, hence, a longer settling time compared to the mp-MPC controllers, but as can be observed in the maintenance phase it will have less undershoot. In Fig. 17, Fig. 18, Fig. 22 and Fig.23 we have the BIS response and the output for patient 9 in the induction phase and the maintenance phase. This patient represents the worst case scenario since it is the most sensitive patient. As we can observe from the figures, all four controllers have good performances and their responses are very close to each other. However, the controller from Case 2 gives the best performances for this patient in the induction phase particularly; lower undershoot, $3.7 \%$ and faster settling time, 300 seconds. This shows that the combination between the linearization method based on gPROMS and optimization methods based on mp-MPC gives good results even without the nonlinearity compensation.

It is important to state that the mp-MPC controller designed using the linearized patient model is the simplest version of the four controllers since it doesn't use an estimator and it avoids using the nonlinearity compensation which introduces additionally complexity in the DOA control. Moreover, it obtains the best performances which can be explained through the fact that the nonlinearity of the Hill curve is more intense at extreme values of the BIS index and weaker around the BIS value of 50 where the model was linearized and where the BIS target is set. If the induction phase and the maintenance phase are kept around the value of 50\%, Case 2 will give very good performances. But if the disturbances take the process out of the 50\% area we can observe that the performances are not as good as in the case of nonlinearity compensation. Case 2 does not provide good performances if the disturbances are substantial. Due to the Hill nonlinearity the real patient model has smaller gain at the extreme values of the control variable. In the case of substantial disturbances, the control variable goes to the extreme values and the controller has a slower response but also a lower undershoot/overshoot.

Using nonlinearity compensation is a good alternative in this case. Moreover, the computations required for the nonlinearity compensation are rather straight forward (the inverse of the Hill curve), and there are no recursive computations that might lead to accumulation of errors.

The estimator used for the mp-MPC with nonlinearity compensation can also be applied for the mp-MPC using local linearization. It was not used for this study because as it can be observed from the simulations the case with nonlinearity compensation is more meaningful in the presence of disturbance.

The aim of the studies on control of anaesthesia is to be able to implement the controllers on embedded devices (see MOBILE project). These types of devices do not have the same computational power as the computers where simulations are performed in real time. This would make classical MPC more difficult to implement since matrix operations are harder to program on embedded devices. The $\mathrm{mp}$ programming algorithms derive the explicit mapping of the optimal control actions as a function of the current states resulting in the implementation of a simple look up table and simple function evaluations. This makes the mp-MPC controllers much easier to implement for the control of depth of anaesthesia.

For each patient there will be a variable dose-response relationship. For the same reference value, the controller sends different drug rate and the blood and effect-site concentrations levels are different for each patient. The safety limit for Propofol blood concentration and effect-site concentration is fulfilled by maintaining the drug infusion rate below $10 \mathrm{mg} / \mathrm{s}$. It can be observed from Fig. 16, Fig. 18, Fig. 21, Fig.23 that the drug infusion rates are maintained below this limit.

Note that the robustness of the performance is analyzed by having the controllers designed on a nominal model [6] and then tested on wide set of patient models parameters where the impact of parameter uncertainties were analyzed. Formal robust criteria can also be included [27] and this represents a topic of our ongoing research.

\section{CONCLUSION}

In this paper we design and evaluate four different controllers for the regulation of depth of anaesthesia during induction and maintenance phase. For the maintenance phase, a realistic disturbance signal was considered and applied. A simulation study is performed on a set of 12 virtually generated patients plus the mean patient. The performance of the four controllers is compared with each other for a sensitive patient and the nominal patient. 
Some important aspects of this application are the high inter-patient variability and the presence of important disturbances during the maintenance phase. The results show a high-efficiency, optimal dosage and robustness of the model predictive control algorithm to induce and maintain the desired Bispectral Index reference while rejecting typical disturbances from surgery. The multi parametric model predictive control approach, which is an offline optimisation method, has similar performances with the online method and promising results.

\section{Acknowledgements}

Financial support from EPSRC (EP/G059071/1, EP/I014640), the European Research Council (MOBILE, ERC Advanced Grant, No: 226462) and the European Commission (OPTICO/G.A. No.280813) is gratefully acknowledged.

\section{REFERENCES}

[1] J. M. Bailey and W. M. Haddad, "Drug dosing control in clinical pharmacology," IEEE Control System Magazine, vol. 25, pp. 3531, 2005.

[2] W. M. Haddad, T. Hayakawa, and J. M. Bailey, "Nonlinear Adaptive Control for Intensive Care Unit Sedation and Operating Room Hypnosis," 2003, pp. 1808-1813.

[3] A. R. Absalom, R. De Keyser, and M. M. R. F. Struys, "Closed loop anaesthesia: are we getting close to finding the Holy Grail?," Anesth Analg, vol. 112, pp. 516-518, 2011.

[4] M. Curatolo, M. Derighetti, S. Petersen-Felix, P. Feigenwinter, M. Fischer, and A. M. Zbinden, "Fuzzy logic control of inspired isoflurane and oxygen concentrations using minimal flow anaesthesia," British Journal of Anaesthesia, vol. 76, pp. 245-250, 1996.

[5] M. M. R. F. Struys, H. Vereecke, A. Moerman, E. W. Jensen, D. Verhaeghen, N. De Neve, et al., "Ability of the Bispectral Index, autoregressive modelling with exogenous input-derived auditory evoked potentials, and predicted propofol concentrations to measure patient responsiveness during anesthesia with propofol and remifentanil," Anesthesiology, vol. 99, pp. 802-812, 2003.

[6] C. M. Ionescu, R. D. Keyser, B. C. Torrico, T. D. Smet, M. M. R. F. Struys, and J. E. Normey-Rico, "Robust predictive control strategy applied for propofol dosing using BIS as a controlled variable during anesthesia," IEEE Transactions on Biomedical Engineering, vol. 55, pp. 2161-2170, 2008.

[7] R. Hodrea, R. Morar, and I. Nascu, "Predictive Control of Neuromuscular Blockade," Automation Computers Applied Mathematics, vol. 21, 2012.

[8] V. Caiado Daniela, M. Lemos João, and A. Costa Bertinho, "Robust control of depth of anesthesia based on Ho design," in Archives of Control Sciences vol. 23, ed, 2013, p. 41.

[9] G. A. Dumont, A. Martinez, and J. M. Ansermino, "Robust control of depth of anesthesia," International Journal of Adaptive Control and Signal Processing, vol. 23, pp. 435-454, 2009.

[10] I. Nascu, I. Nascu, C. M. Ionescu, and R. De Keyser, "Adaptive EPSAC predictive control of the hypnotic component in anesthesia," 2012, pp. 103-108.

[11] I. Nascu, R. S. C. Lambert, A. Krieger, and E. N. Pistikopoulos, "Simultaneous multi-parametric model predictive control and state estimation with application to distillation column and intravenous anaesthesia," in Computer Aided Chemical Engineering vol. 33, ed, 2014, pp. 541-546.

[12] A. Krieger, N. Panoskaltsis, A. Mantalaris, M. C. Georgiadis, and E. N. Pistikopoulos, "Analysis of an individualized physiologically based model for anesthesia control," 2012, pp. 385-390.

[13] A. Krieger, N. Panoskaltsis, A. Mantalaris, M. C. Georgiadis, and E. N. Pistikopoulos, "Modeling and analysis of individualized pharmacokinetics and pharmacodynamics for volatile anesthesia," IEEE Transactions on Biomedical Engineering, vol. 61, pp. 25-34, 2014.
[14] C. M. Ionescu, I. Nascu, and R. Keyser, "Robustness Tests of a Model Based Predictive Control Strategy for Depth of Anesthesia Regulation in a Propofol to Bispectral Index Framework," in International Conference on Advancements of Medicine and Health Care through Technology. vol. 36, S. Vlad and R. Ciupa, Eds., ed: Springer Berlin Heidelberg, 2011, pp. 234-239.

[15] I. Nascu, C. M. Ionescu, I. Nescu, and R. De Keyser, "Evaluation of three protocols for automatic DOA regulation using Propofol and Remifentanil," 2011, pp. 573-578.

[16] C. M. Ionescu, I. Nascu, and R. De Keyser, "Towards a multivariable model for controlling the depth of anaesthesia using propofol and Remifentanil," 2012, pp. 325-330.

[17] M. M. R. F. Struys, T. De Smet, S. Greenwald, A. R. Absalom, S. Bingé, and E. P. Mortier, "Performance Evaluation of Two Published Closed-loop Control Systems Using Bispectral Index Monitoring: A Simulation Study," Anesthesiology, vol. 100, pp. 640-647, 2004.

[18] T. W. Schnider, C. F. Minto, P. L. Gambus, C. Andresen, D. B. Goodale, S. L. Shafer, et al., "The influence of method of administration and covariates on the pharmacokinetics of propofol in adult volunteers," Anesthesiology, vol. 88, pp. 1170-1182, 1998. T. W. Schnider, C. F. Minto, S. L. Shafer, P. L. Gambus, C. Andresen, D. B. Goodale, et al., "The influence of age on propofol pharmacodynamics," Anesthesiology, vol. 90, pp. 1502-1516, 1999.

[20] C. F. Minto, T. W. Schnider, and S. L. Shafer, "Pharmacokinetics and pharmacodynamics of remifentanil. II. Model application," Anesthesiology, vol. 86, pp. 24-33, Jan 1997.

[21] C. F. Minto, T. W. Schnider, T. D. Egan, E. J. Youngs, H. J. Lemmens, P. L. Gambus, et al., "Influence of age and gender on the pharmacokinetics and pharmacodynamics of reminfetanil. I. Model development," Anesthesiology, vol. 86, pp. 10-23, 1997.

[22] N. West, G. A. Dumont, K. Van Heusden, C. L. Petersen, S. Khosravi, K. Soltesz, et al., "Robust closed-loop control of induction and maintenance of propofol anesthesia in children," Paediatric Anaesthesia, vol. 23, pp. 712-719, 2013.

[23] C. S. Nunes, T. Mendonça, J. M. Lemos, and P. Amorim, "Feedforward adaptive control of the Bispectral Index of the EEG using the intravenous anaesthetic drug propofol," International Journal of Adaptive Control and Signal Processing, vol. 23, pp. 485-503, 2009.

[24] C. Ionescu, J. T. Machado, R. De Keyser, J. Decruyenaere, and M. M. R. F. Struys, "Nonlinear dynamics of the patient's response to drug effect during general anesthesia," Communications in Nonlinear Science and Numerical Simulation.

[25] R. De Keyser, "Model Based Predictive Control " Invited Chapter in UNESCO Encyclopaedia of Life Support Systems (EoLSS), vol. 6.43.16.1, p. 30p, 2003.

[26] E. N. Pistikopoulos, M. Georgiadis, and V. Dua, "Multi-parametric Programming: Theory, Algorithms and Applications," Wiley-VCH, 2007 a.

[27] K. I. Kouramas, N. P. Faísca, C. Panos, and E. N. Pistikopoulos, "Explicit/multi-parametric model predictive control (MPC) of linear discrete-time systems by dynamic and multi-parametric programming," Automatica, vol. 47, pp. 1638-1645, 2011.

[28] P. S. E. L. (PSE), "gPROMS Model Developer Guide," Release v3.3.0, vol. http://www.psenterprise.com/gproms/, 2010.

[29] D. W. Clarke, C. Mohtadi, and P. Tuffs, "Generalized predictive control-Part I. The basic algorithm," Automatica, vol. 23, pp. 137-148, 1987.

[30] C. Mohtadi, D. Clarke, and P. Tuffs, "Generalized predictive control-part II. Extensions and Interpretations," Automatica, vol. 23, pp. 149-160, 1987.

[31] G. F. Franklin, D. J. Powell, and A. Emami-Naeini, Feedback Control of Dynamic Systems: Prentice Hall PTR, 2001.

[32] E. N. Pistikopoulos, N. A. Bozinis, and V. Dua, "POP: A Matlab implementation of multi-parametric quadratic programming algorithm," London, UK: Centre for Process System Engineering, Imperial College, 1999.

[33] G. Welch and G. Bishop, "An introduction to the Kalman filter," Course at SIGGRAPH.

[34] S. Yelneedi, L. Samavedham, and G. P. Rangaiah, "Advanced Control Strategies for the Regulation of Hypnosis with Propofol," Industrial \& Engineering Chemistry Research, vol. 48, pp. 3880$3897,2009 / 04 / 152009$. 